\section{Possible roles of transglutami- nases in molecular mechanisms responsible for cancer and human neurodegenerative diseases}

\author{
Gabriella Misso, Nicola Gaetano Gatta, \\ Mayra Rachele Zarone, \\ Gaetano Cammarota, Anna Grimaldi, \\ Vittorio Gentile \\ Department of Biochemistry, Biophysics \\ and General Pathology, Second \\ University of Naples, Naples, Italy
}

\begin{abstract}
Transglutaminases are a family of $\mathrm{Ca}^{2+}-$ dependent enzymes which catalyze posttranslational modifications of proteins. The main activity of these enzymes is the crosslinking of glutaminyl residues of a protein/peptide substrate to lysyl residues of a protein/peptide co-substrate. In addition to lysyl residues, other second nucleophilic co-substrates may include monoamines or polyamines (to form mono- or bi-substituted/crosslinked adducts) or -OH groups (to form ester linkages). In absence of co-substrates, the nucleophile may be water, resulting in the net deamidation of the glutaminyl residue. Transglutaminase activity has been suggested to be involved in molecular mechanisms responsible for either physiological or pathological processes. In particular, transglutaminase activity has been shown to be responsible for human autoimmune diseases and Celiac Disease is just one of them. Interestingly, cancer and neurodegenerative diseases, such as Alzheimer's Disease, Parkinson's Disease, supranuclear palsy and Huntington's Disease, are characterized in part by aberrant transglutaminase activity and by increased cross-linked proteins in affected tissues. This review describes the possible molecular mechanisms by which these enzymes could be responsible for such diseases and the possible use of transglutaminase inhibitors for patients with diseases characterized by aberrant transglutaminase activity.
\end{abstract}

\section{Biochemistry of the transglutam- inases}

Transglutaminases (TGs, E.C. 2.3.2.13) are family of $\mathrm{Ca}^{2+}$-dependent enzymes which catalyze post-translational modifica- tions of proteins. Examples of TG-catalyzed reactions include: I) acyl transfer between the $\gamma$-carboxamide group of a protein/polypeptide glutaminyl residue and the $\varepsilon$-amino group of a protein/polypeptide lysyl residue; II) attachment of a polyamine to the $\gamma$-carboxamide of a glutaminyl residue; III) deamidation of the $\gamma$-carboxamide group of a protein/polypeptide glutaminyl residue. ${ }^{1,2}$ The reactions catalyzed by TGs occur by a two-step mechanism (pingpong type). The transamidating activity of TGs is activated by the binding of $\mathrm{Ca}^{2+}$, which exposes an active-site cysteine residue. This cysteine residue reacts with the $\gamma$-carboxamide group of an incoming glutaminyl residue of a protein/peptide substrate to yield a thioacyl-enzyme intermediate and ammonia. The thioacyl-enzyme intermediate then reacts with a nucleophilic primary amine substrate, resulting in the covalent attachment of the amine-containing donor to the substrate glutaminyl acceptor and regeneration of the cysteinyl residue at the active site. If the primary amine is donated by the $\varepsilon$-amino group of a lysyl residue in a protein/polypeptide, a $\mathrm{N}^{\varepsilon}-(\gamma-\mathrm{L}-$ glutamyl)-L-lysine (GGEL) isopeptide bond is formed. On the other hand, if a polyamine or another primary amine (e.g. histamine, serotonin and others) acts as the amine donor, a $\gamma$-glutamylpolyamine (or $\gamma$ glutamylamine) residue is formed. It is also possible for a polyamine to act as an N,Nbis-( $\gamma$-L-glutamyl)polyamine bridge between two glutaminyl acceptor residues either on the same protein/polypeptide or between two proteins/polypeptides. ${ }^{3}$ If there is no primary amine present, water may act as the attacking nucleophile, resulting in the deamidation of glutaminyl residues to glutamyl residues. Regarding the physiological roles played by the transglutaminase activity, recently transglutaminase-catalyzed polyamination of tubulin has been shown to stabilize axonal microtubules, suggesting an important role for these reactions also during some physiological processes, such as neurite outgrowth and axon maturation. ${ }^{4}$ The reactions catalyzed by TGs occur with little change in free energy and hence should theoretically be reversible. However, under physiological conditions the cross linking reactions catalyzed by TGs are usually irreversible. This irreversibility partly results from the metabolic removal of ammonia from the system and from thermodynamic considerations resulting from altered protein conformation. Some scientific reports suggest that TGs may be able to catalyze the hydrolysis of GGEL cross links isopeptide bonds in some soluble crosslinked proteins. Furthermore, it is likely that TGs can catalyze the exchange of
Correspondence: Gabriella Misso, Department of Biochemistry, Biophysics and General Pathology, University of Campania "Luigi Vanvitelli", via L. de Crecchio 7, 80138 Naples, Italy.

Phone: +39 081 5665874; Fax: +390815665863.

E-mail: gabrymisso@yahoo.it

Vittorio Gentile, Department of Biochemistry, Biophysics and General Pathology, University of Campania "Luigi Vanvitelli", via L. de Crecchio 7, 80138 Naples, Italy.

Phone: +39081 5665870; Fax: +39081 450169 .

E-mail: vittorio.gentile@unicampania.it

Key words: Transglutaminases; Post-translational modifications of proteins; Cancer; NF$\mathrm{kB}$; Neurodegeneration.

Contributions: N.G.G., G.C., M.R.Z. and A.G. selected studies and collected data from literature; V.G., and G.M. prepared the manuscript; N.G.G., G.C., M.R.Z. and A.G. implemented and edited the manuscript; V.G. and G.M. critically revised the manuscript; V.G. finally approved the manuscript.

Funding: this work is supported by the Italian Education Department and the Campania Region (L.R. n.5 del 28.03.2002, finanziamento 2008) entitled: Identificazione e caratterizzazione di geni della transglutaminasi nel Sistema Nervoso in relazione allo sviluppo di malattie neurodegenerative (Identification and characterization of transglutaminase genes in the Nervous System in relationship to the development of neurodegenerative diseases).

Conflict of interest: the authors declare no potential conflict of interest.

Received for publication: 26 January 2017. Accepted for publication: 24 February 2017.

This work is licensed under a Creative

polyamines onto proteins. ${ }^{2}$ In TG2 other catalytic activities, such as the ability to hydrolyze GTP (or ATP) into GDP (or ADP) and inorganic phosphate, a protein disulfide isomerase activity and a kinase activity which phosphorylates histones, retinoblastoma (RB) and P53 are present, while only some of these activities have been identified also in other TGs. ${ }^{5-8}$

Ample experimental evidence indicate that some TGs are multifunctional proteins with distinct and regulated enzymatic activities. In fact, under physiological conditions, the transamidation activity of TGs is latent, 9,10 while other activities, recently identified, could be present. For example, in some physiological states, when the concentration of $\mathrm{Ca}^{2+}$ increases, the crosslinking activity of TGs may contribute to important biological processes. As previously described, one of the most intriguing 
properties of some TGs, such as TG2, is the ability to bind and hydrolyze GTP and furthermore, to bind to GTP and $\mathrm{Ca}^{2+}$. GTP and $\mathrm{Ca}^{2+}$ regulate its enzymatic activities, including protein cross-linking, in a reciprocal manner: the binding of $\mathrm{Ca}^{2+}$ inhibits GTP-binding and GTP-binding inhibits the transglutaminase cross-linking activity of the TG2.5 Interestingly, TG2 shows no sequence homology with heterotrimeric or low-molecular-weight G-proteins, but there is evidence that TG2 (TG2/Gh $\alpha)$ is involved in signal transduction, and, therefore, $\mathrm{TG} 2 / \mathrm{Gh} \alpha$ should also be classified as a large molecular weight G-protein. Other studies, along with ours, showed that TG2/Gh $\alpha$ can mediate the activation of phospholipase C (PLC) by the $\alpha_{1 b}$-adrenergic receptor ${ }^{10}$ and can modulate adenylyl cyclase activity. ${ }^{11}$ TG2/Gh $\alpha$ can also mediate the activation of the $\delta 1$ isoform of PLC and of maxi-K channels. ${ }^{12}$ Interestingly, the signaling function of $\mathrm{TG} 2 / \mathrm{Gh} \alpha$ is preserved even with the mutagenic inactivation of its crosslinking activity by the mutation of the active site cysteine residue. ${ }^{13}$

\section{Molecular biology of the transg- lutaminases}

To date at least eight different TGs, distributed in the human body, have been identified (Table 1). ${ }^{14-19}$ Complex gene expression mechanisms regulate the physiological roles that these enzymes play in both the intracellular and extracellular compartments. In the Nervous System, for example, several forms of TGs are simultaneously expressed. ${ }^{20-22}$ Moreover, in these last years, several alternative splice variants of TGs, mostly in the 3'-end region, have been identified. ${ }^{23}$ Interestingly, some of them are differently expressed in human pathologies, such as Alzheimer's Disease (AD). ${ }^{24}$ On the basis of their ubiquitous expression and their biological roles, we may speculate that the absence of these enzymes would be lethal. However, this does not always seem to be the case, since, for example, null mutants of the TG2 are usually phenotypically normal at birth. ${ }^{12,25,26}$ This result may be explained by the expression of other TG genes that may substitute the TG2 missing isoform, although other TG isoform mutations have been associated with severe phenotypes, such as lamellar ichthyosis for TG1 isoform mutations. Bioinformatic studies have shown that the primary structures of human TGs share some identities in only few regions, such as the active site and the calcium binding regions. However, high sequence conservation and, therefore, a high degree of preservation of secondary structure among TG2, TG3 and FXIIIa indicate that these TGs all share four-domain tertiary structures which could be similar to those of other TGs. ${ }^{27}$

\section{Transglutaminases and signal transduction}

Beside the well-known function as a crosslinking enzyme, TG2 is able to interact with different target proteins dislocated in extracellular matrix, membrane, cytoplasm, mitochondrion and nucleus. ${ }^{27}$ This fact implies that TG2 can work as GTPase/ATPase, non-enzymatic adapter, scaffold protein or signal transduction regulator in processes such as transamidation and protein-protein crosslinking. Among the most characterized transglutaminaseregulated cell signaling there is the pathway of TGF- $\beta$, whose maturation and activity is induced by TG2 through a covalent crosslink with latent TGF- $\beta 1$ binding protein. ${ }^{28}$ Change in growth factor activity results in extracellular environment remodeling ${ }^{29}$ involving integrins and proteases. Furthermore, in tumor models, TG2 expression was reported to be induced by TGF- $\beta$, resulting in the induction of epithelial-tomesenchymal transition (EMT), an embryonic process that can be reactivated in adult tissues in response to epigenetic changes. ${ }^{30}$ In turn, a mechanism involving NF-kB pathway is responsible for the increase, in fibroblasts, of both TGF- $\beta$ mRNA and protein expression. ${ }^{31}$ Therefore TG2 and TGF$\beta$ are reciprocally regulated in a positive feedback loop. TG2 acts, in fact, also as a $\mathrm{NF}-\kappa \mathrm{B}$ activator in a IKK-independent mechanism that involves TG2 crosslinking of $\mathrm{I} \kappa \mathrm{B} \alpha^{32}$ with subsequent I $\mathrm{K} \mathrm{B} \alpha$ degradation via a non-proteasomal mechanism. TG2 also promotes polymerization of $\mathrm{I} \kappa \mathrm{B} \alpha$ that induces $\mathrm{I} \kappa \mathrm{B} \alpha$ proteasomal degradation. ${ }^{32}$ Both mechanisms lead to NF- $\kappa \mathrm{B}$ activation. In addition, TG2 contributes to cell attachment to the matrix, activating integrin signaling that is achieved by TG2 direct binding with $\beta 1, \beta 3$ and $\beta 5$ integrins. ${ }^{33} \mathrm{~A}$ strong interaction also affects TG2 and fibronectin. Whereby it occurs a dual binding both with both fibronectin and integrin, that potentiates integrin/fibronectin interaction. More recently, it has been identified a role for TG2 in the interplay between integrin and platelet-derived growth factor receptor (PDGFR). This interaction enhances PDGFR binding with integrins ${ }^{34,35}$ thus upregulating growth factor receptor downstream signalling and PDGFR turnover. It results in the stimulation of PDGF/PDGFR- induced Akt1 and Shp2 activation in fibroblasts and vascular smooth muscle cells, ${ }^{34}$ enhancing cell proliferation, survival and migration. TG2 also affects VEGF pathway by covalently binding VEGFR on the surface of endothelial cells, ${ }^{36}$ so originating a high-molecular-weight complex that heads toward the nucleus and potentiates VEGFinduced ERK activation. ${ }^{36}$ Also EGFR pathway, that is commonly aberrantly activated in cancer, induces the expression of TG2 in epithelial tumor cells; ${ }^{37}$ this is in direct correlation with the increase of TG2-dependent transamidation and with the subsequent activation EGF-dependent signalling involving Ras, PI3K, JNK, ERK and NF- $\kappa \mathrm{B}$ pathways. ${ }^{37}$ As a result of EGF-mediated TG2 induction, it can be observed enhanced migration, invasiveness and anchorageindependent cancer cell growth. ${ }^{37}$

Of note, TG2 mediates monoaminylation of cytoplasmic proteins regulating signaling and vesicular trafficking; among these post-translational modifications, there is the serotonylation of RhoA and Rab4A GTPases, essential for cytoskeletal reorganization and subsequent exocytosis of platelet $\alpha$-granules, platelet activation, platelet adhesion, and platelet aggregation. ${ }^{38}$ RhoA activation, through TG2-mediated monoaminylation, is also correlated with Aktlactivation. ${ }^{39}$ Another post-translational modification triggered by transglutaminase is the eIF5A-1 hypusination, as demonstrated by the in vitro formation of a $\gamma$-glutamyl-hypusine bond among eIF5A-1 and dimethylcasein. ${ }^{40}$ This event was put in correlation with the inhibition of cell proliferation and apoptosis induction. On the other hand, it was reported that IFN $\alpha$ reduced the hypusination and activation of eIF5A-1 in parallel with the induction of growth inhibition in human epidermoid cancer KB cells and all these effects were antagonized by EGF. ${ }^{41,42}$ Concerning type I IFN, and particularly IFN $\beta$, it was report$\mathrm{ed}^{43,44}$ that it can stimulate the transcription of TG2, although the precise mechanism has not been wholly elucidated. Moreover, IFN can also affect TG intracellular stability acting on its ubiquitination, maybe through the induction of two ubiquitin cross-reactive enzymes: $\mathrm{UbcH5}$ and $\mathrm{UbcH} 8 .{ }^{45}$ Similarly, also IFN $\alpha$ has been demonstrated stimulatory activity over TG2 expression in a global anticancer mechanism that involves the inhibition, IFN $\alpha$-mediated, of TG2 degradation via the ubiquitin proteasome system. ${ }^{46}$ Regardless of transamidating activity, TG2 also interacts with GTP and acts as GTPase, in spite of the lack of four consensus GTP-binding motifs common to the classical $G$ proteins. The catalytic rate is comparable to the $\alpha$ subunits of $G$ 
proteins and mutating Arg580 accounts for an about 100-fold decrease in GTP-binding affinity and deletes the inhibitory activity of GTP against TG2-mediated transamidation. ${ }^{47}$ TG2-mediated protein crosslinking, transamidation and GTPase activity are also realized in nuclear compartment, where it is localized an about 5\% of TG2 total cellular amount. ${ }^{48}$ Nuclear TG2 is involved in chromatin binding and, moreover, in the regulation of gene expression through the transamidation of both histones and transcription factors, such as Sp1, which is inactivated by TG2 crosslink, with subsequent downregulation of growth factor receptors, including c-Met, and cell death. ${ }^{49}$ Finally, TG2 is also present in both mitochondrial membrane and mitochondrial matrix; ${ }^{48,50}$ a sequence omology with $\mathrm{BH} 3$ domain of Bcl-2 mitocondrial proteins, indicates an apoptotic role ${ }^{50}$ that can be pro- or anti-apoptotic, depending on the cell type and on the precise death stimulus. For instance, TG2 can crosslink the proapoptotic members inducing cell death in certain cell models and, on the contrary, it can inhibits calcium-induced apoptosis in other cell types. ${ }^{51}$ Moreover, several reports have also shown TG2-induced transamidation and crosslinking of mitochondrial proteins, although it seems that these modifications are a peculiarity of not normal tissues, such as the ones affected by mitochondrial diseases, i.e. those suffering from cardiovascular or neurodegenerative disorders. ${ }^{52}$ Overall, these results support the hypothesis that increased TG2 expression levels are able to promote proliferative pathways both by its binding with key signaling proteins and by acting on the extracellular matrix.

\section{Transglutaminases and cancer}

Among the best characterized physiological roles of TG2 there is the regulation of several processes, such as cell growth and differentiation, ${ }^{53}$ tumor metastasis and programmed cell death. ${ }^{54,55}$ Aside from genetic and epigenetic changes, that can be induced by several stressor agents, a strong contribute in dysregulating cell growth and differentiation seems to be due to chronic inflammation. It gives, in fact, a further effort in conferring metastatic potential and capability to survive in stromal environment, thus contributing to cancer initiation and progression. ${ }^{56}$ Assuming that TG2 is commonly upregulated in inflamed tissues,${ }^{57}$ it is expected that TG2 could be involved in cancer development, although the molecular mechanisms remain mostly to be delucidated. Anyway, it has been demon- strated that TG2 expression is low in primary tumors and higher in drug-resistant cancers and metastatic tissues. ${ }^{58,59}$ The correlation between TG2 and inflammation that, on the basis of what has just been stated, establish an indirect correlation between TG2 and cancer, has been documented by the fact that the common inflammatory cytokines (TGF- $\beta$, TNF- $\alpha$, IL- 1 , and IL-6) are well-known TG2 stimulators ${ }^{60,61}$ and are able to establish an active autocrine stimulation in tumor environment. Cytokineinduced increase of TG2 levels stimulates both synthesis and deposition of fibronectin and collagen; simultaneously, extracellular TG2 crosslinks them and stabilizes the extracellular matrix, thereby inducing tumor desmoplastic response ${ }^{62}$ and promoting malignant phenotype. As previously stated, TG2-dependent activation of NF- $\kappa B$ signalling, as well as also FAK and Akt stimulation, make it a strong EMT inducer. ${ }^{63,64}$ EMT controls cell behavior during cancer progression in an effort to hold inflammation and repair damaged tissues; nevertheless, in pathological tissues this response can account for damage, resulting in intravasation, extravasation and micrometastases formation and the acquisition of stem-cell phenotype. ${ }^{63,65,66}$ Furthermore, hypoxia-response transcription factor HIF-1, whose expression is controlled by $N F-\kappa B^{67}$ and TG2, ${ }^{63,65,66}$ has itself an important role in cancer development and in inflammatory signaling regulation, controlling EMT, stemness, drug resistance and metastatic phenotype. In fact, cells expressing high TG2 levels exhibit elevated HIF-1 $\alpha$ levels, even under normoxic conditions; on the other hand, enforced inhibition of either TG2 or NF- $\kappa$ B induces a decrease of HIF$1 \alpha$ expression. ${ }^{68}$ Of note, the covalent modification of basement membrane proteins (laminin, collagen and diverse types of extracellular matrix proteins) by TG may interfere with the adhesiveness and invasiveness of tumor cells, ${ }^{69,70}$ thus further supporting the participation of this enzyme in the migration and adhesion processes occurring during the metastatic spread of cancer cells. TG2 catalyzes the incorporation of spermidine into the components of basal membrane, in a process that generally involves FAD-dependent polyamine oxidase $(\mathrm{PAO})^{71}$ and that may be one of the cellular mechanisms regulating the preferential formation of a sterically defined bis( $\gamma$-glutamyl)spermidine cross-link. In virtue of this fact, it was investigated the effect of this catalytic activity on the adhesion and invasion capability of murine B16-F10 melanoma cells. The analysis was carried out using laminine or matrigel as reference components of basal membrane. In virtue of the impairment of extracellular matrix crosslinking, adhesiveness of B16-F10 cells to basal membrane proteins become reduced when the basal-like proteins were modified by spermidine, due to TG2 catalysis. This is of interest taking into account that the adhesion of circulating tumor cells to endothelium and their transition through the vascular endothelium is a main stage in cancer invasion and metastasis. ${ }^{72,73}$ Concerning the previously hinted TG2 involvement in drug resistance, a recent report has described the correlation between TG2 and human MCF-7 breast cancer cells' resistance to vorinostat, as well as to other histone deacetylase (HDAC) inhibitors. TG2 mRNA and protein expression levels, along with TG2 transamidating activity, were paralleled with vorinostat-induced anti-proliferative effects, founding an inverse correlation and suggesting that TG2 could represent a mechanism of intrinsic or acquired resistance to vorinostat. ${ }^{74}$ Similar results have also been observed for $\mathrm{MCF}-7$ cells treated with doxorubicin, where there was a selection of a small cell subpopulation with stemness characteristics and high TG2 expression levels. ${ }^{75}$ All these findings strongly suggest that TG2 can promote the EMT and is also responsible for the induction of a stem cell behaviour in tumor cells.

\section{Transglutaminases as target for therapeutic intervention in cancer}

Drug resistance and metastasization are features closely related to an advanced stage (relapsed and refractory) of cancer and represent the most clinical obstacle to the success of anti-cancer treatment, accounting for about $90 \%$ of cancer-related deaths. The discovery of tumor-associated genes able to induce metastasization and resistance to anti-cancer therapy is an imperative objective in order to properly target them and to employ the corresponding proteins as cancer biomarkers. Based on the pleiotropic characteristics of TG2 and on the finding of higher TG2 expression levels in tumor samples, compared to the normal counterpart, ${ }^{76-78}$ it was widely investigated the role played by TG2 in inducing cancer hallmarks. TG2 inhibition, employing siRNA, antisense RNA or small molecule inhibitors, should represent a promising therapeutic option for treating advanced cancers and counteracting entire inflammatory networks $(\mathrm{NF}-\kappa \mathrm{B}, \mathrm{AKT}$, FAK, HIF-1 $\alpha$, etc.) that play a crucial role in inducing angiogenesis, drug resistance, metastasization and EMT. Targeted thera- 
pies affecting a single gene or pathway are often responsible for the occurrence of resistance, therefore it is widely desirable an anti-cancer strategy based on the inhibition of multiple signalling pathways that work in parallel in cancer cells. This can be achieved either by multi-drug therapy or by multi-targeting agents, such as TG2 inhibitors. ${ }^{79,80}$ To date, several small TG2 inhibitors have been developed. ${ }^{81}$ However, if on one hand some of these have shown promising in vitro and in vivo activity, their clinical use still presents serious limitations. ${ }^{82}$ TGs are complex proteins with various structural domains bearing different functions; therefore, it is difficult to target them using small molecules or antibodies that, among other things, often lack specificity and may originate undesirable side effects. In this regard, siRNA treatment to inhibit TG2 expression remains the most useful approach for cancer cells treatment. Liposomal delivery of siRNA has been successfully employed in orthotopically growing tumors, showing a significant downregulation of TG2 expression. ${ }^{58,83}$ This delivery strategy resulted in in vivo TG2 silencing as well as in the inhibition of growth and dissemination of pancreatic and ovarian cancer cells, also rendering them sensitive to chemotherapeutic drugs in a nude mouse model. ${ }^{84,85}$ These results endorse the hypothesis that high TG2 levels may not only be used as reliable prognostic markers for early tumor diagnosis but also as promising therapeutic target for treating advanced tumors.

\section{Role of the transglutaminases in neurodegenerative diseases}

Although numerous scientific reports suggest that the transglutaminase activity is involved in the pathogenesis of neurodegenerative diseases, to date, however, still controversial experimental findings about the role of the TGs enzymes in these diseases have been obtained. ${ }^{86-88}$ Protein aggregates in affected brain regions are histopathological hallmarks of many neurodegenerative diseases. ${ }^{89}$ More than 20 years ago Selkoe et al. ${ }^{90}$ suggested that TG activity might contribute to the formation of protein aggregates in $\mathrm{AD}$ brain. In support of this hypothesis, tau protein has been shown to be an excellent in vitro substrate of TGs ${ }^{91,92}$ and GGEL cross-links have been found in the neurofibrillary tangles and paired helical filaments of $\mathrm{AD}$ brains. ${ }^{93}$ Interestingly, a recent work showed the presence of bis $\gamma$-glutamyl putrescine in human CSF, which was increased in Huntington's Disease (HD) CSF. ${ }^{94}$ This is an important evidence that protein/peptides crosslinking by polyamines does indeed occur in the brain, and that this is increased in HD brain. TG activity has been shown to induce also amyloid $\beta$-protein oligomerization $^{95}$ and aggregation at physiologic levels. ${ }^{96}$ By these molecular mechanisms, TGs could contribute to AD symptoms and progression. ${ }^{96}$ Moreover, there is evidence that TGs also contribute to the formation of proteinaceous deposits in Parkinson's Disease (PD), ${ }^{97,98}$ in supranuclear palsy ${ }^{99,100}$ and in $\mathrm{HD}$, a neurodegenerative disease caused by a CAG expansion in the affected gene. ${ }^{101}$ For example, expanded polyglutamine domains have been reported to be substrates of TG2 $2^{102-104}$ and therefore aberrant TG activity could contribute to CAGexpansion diseases, including HD. However, although all these studies suggest the possible involvement of the TGs in the formation of deposits of protein aggregates in neurodegenerative diseases, they do not indicate whether aberrant TG activity per se directly determines the disease progression. For example, several experimental findings reported that TG2 activity in vitro leads to the formation of soluble aggregates of $\alpha$ synuclein ${ }^{105}$ or polyQ proteins. ${ }^{106,107}$ To date, as previously reported, at least ten human CAG-expansion diseases have been described (Table 2) ${ }^{108-117}$ and in at least eight of them their neuropathology is caused by the expansion in the number of residues in the polyglutamine domain to a value beyond 35-40. Remarkably, the mutated proteins have no obvious similarities except for the expanded polyglutamine domain. In fact, in all cases except SCA 12, the mutation occurs in the coding region of the gene. However, in SCA12, the CAG triplet expansion occurs in the untranslated region at the 5' end of the PPP2R2B gene. It has been proposed that the toxicity results from overexpression of the brain specific regulatory subunit of protein phosphatase PP2A. ${ }^{114}$ Most of the mutated proteins are widely expressed both within the brain and elsewhere in the body. A major challenge then is to understand why the brain is primarily affected and why different regions within the brain are affected in the different CAG-expansion diseases, i.e., what accounts for the neurotoxic gain of function of each protein and for a selective vulnerability of each cell type. Possibly, the selective vulnerability ${ }^{118}$ may be explained in part by the susceptibility of the expanded polyglutamine domains in the various CAG-expansion diseases to act as cosubstrates for a brain TG. To strengthen the possible central role of the TGs in neurodegenerative diseases, a study by Hadjivassiliou et al. ${ }^{119}$ showed that antiTG2 IgA antibodies are present in the gut and brain of patients with gluten ataxia, a non-genetic sporadic cerebellar ataxia, but not in ataxia control patients. Recently, antiTG2, -TG3 and -TG6 antibodies have been found in sera from CD patients, suggesting a possible involvement also of other TGs in the pathogenesis of dermatitis herpetiformis and gluten ataxia, two frequent extra intestinal manifestations of gluten sensitivity. ${ }^{120,121}$ These last findings could suggest also a possible role of the gut-brain axis for the etiopathogenesis of human neurodegenerative diseases, in which the TG enzymes, in particular the TG2 enzyme, could play an important role. ${ }^{122-124}$

In support of the hypothesis of the toxic effect of TG activity in other neurodegenerative diseases, such as Alzheimer's disease and Parkinson's Disease, TG activity has been shown to induce amyloid beta-protein and $\alpha$-synuclein oligomerization and aggre-

Table 1. Transglutaminases and their physiological roles when known.

\begin{tabular}{llcc} 
TIG & Physiological role & Gene map location & Reference \\
Factor XIIla & Blood clotting & $6 \mathrm{p} 24-25$ & 14 \\
TG 1 (Keratinocyte TG, kTG) & Skin differentiation & $14 q 11.2$ & 15 \\
\hline TG 2 (Tissue TG, tTG, cTG) & Apoptosis, cell adhesion, signal transduction & $20 \mathrm{q} 11-12$ & 16 \\
TG 3 (Epidermal TG, eTG) & Hair follicle differentiation & $20 \mathrm{p} 11.2$ & 17 \\
\hline TG 4 (Prostate TG, pTG) & Suppression of sperm immunogenicity & $3 q 21-2$ & 18 \\
TG 5 (TG X) & Epidermal differentiation & $15 q 15.2$ & 19 \\
TG 6 (TG Y) & Central Nervous System development & 20 p13 & 19 \\
TG 7 (TG Z) & Unknown function & $15 q 15.2$ & 19 \\
\hline
\end{tabular}


gation at physiologic levels. ${ }^{125-127}$ In fact, TG activity induces protofibril-like amyloid beta-protein assemblies that are protease-resistant and inhibit long-term potentiation. ${ }^{96}$ Therefore, by these molecular mechanisms, TG activity could also contribute to Alzheimer's disease symptoms and progression. Very recently, TG2 and its isopeptide product have been found increased in Alzheimer's disease and APPswe/PS1dE9 double transgenic mice brains, ${ }^{128}$ while catalytically active TG2 colocalizes with $\mathrm{A} \beta$ pathology in Alzheimer's disease mouse models. ${ }^{129}$ Interestingly, other works are suggesting that also other TGs could be involved in the molecular mechanisms responsible for neurodegenerative diseases. ${ }^{130}$ In particular, a recent work by Basso et al. ${ }^{131}$ found that in addition to TG2, TG1 gene expression level is significantly induced following stroke in vivo or due to oxidative stress in vitro. Moreover, structurally diverse inhibitors, used at concentrations that simultaneously inhibit TG1 and TG2 are neuroprotective. Together, these last studies suggested that multiple TG isoforms, not only TG2, participate in oxidative stress-induced cell death signalling, and that isoform nonselective inhibitors of TG will be most efficacious in combating oxidative death in neurological disorders. These are interesting and worthwhile studies, suggesting that multiple TG isoforms can participate in neuronal death processes. Therefore, all these studies suggest that the involvement of brain TGs could represent a common denominator in several neurological diseases, which can lead to the determination of pathophysio- logical consequences through different molecular mechanisms.

\section{Role of the transglutaminase activity in neuroinflammation}

Neuroinflammation plays an important role in various chronic neurodegenerative diseases, characterized also by the pathological accumulation of specific protein aggregates. In particular, several of these proteins have been shown to be substrates of transglutaminases. Interestingly, it has recently been demonstrated that transglutaminase 2 (TG2) may also be involved in molecular mechanisms underlying inflammation. In the central nervous system, astrocytes and microglia are the cell types mainly involved in this inflammatory process. The transcription factor $\mathrm{NF}-\kappa \mathrm{B}$ is considered the main regulator of inflammation and it is activated by a variety of stimuli including calcium influx, oxidative stress and inflammatory cytokines. Recently, in addition to these stimuli, TG2 has been shown to activate NF- $\mathrm{KB}$ both via a canonical pathway ${ }^{132}$ and via a non-canonical pathway. ${ }^{133}$ On the other hand, NF- $\mathrm{BB}$ regulatory response elements are present also in the TG2 promoter. ${ }^{134}$ Under these conditions, the over-expression of TG2 results in the sustained activation of NF- $\kappa$ B. Several findings emphasize the possible role of the $\mathrm{TG} 2 / \mathrm{NF}-\kappa \mathrm{B}$ activation pathway in neurodegenerative diseases, including Alzheimer's disease, Parkinson's disease, multiple sclerosis and amyotrophic lateral sclerosis.
Together, these evidences suggest that TG2 could play a role in neuroinflammation and could contribute to the production of compounds that are potentially deleterious to neuronal cells. ${ }^{135}$

\section{Transglutaminase inhibition as possible therapeutic approach in neurodegenerative diseases}

In consideration to the fact that up to now there have been no long-term effective treatments for the human neurodegenerative diseases previously reported, then the possibility that selective TG inhibitors may be of clinical benefit has been seriously considered. In this respect, some encouraging results have been obtained with $\mathrm{TG}$ inhibitors in preliminary studies with different biological models of CAG-expansion diseases. For example, cystamine is a potent in vitro inhibitor of enzymes that require an unmodified cysteine at the active site. ${ }^{136}$ Inasmuch as TGs contain a crucial activesite cysteine, cystamine has the potential to inhibit these enzymes by a sulfide-disulfide interchange reaction. A sulfide-disulfide interchange reaction results in the formation of cysteamine and a cysteamine-cysteine mixed disulfide residue at the active site. Recent studies have shown that cystamine decreases the number of protein inclusions in transfected cells expressing the atrophin (DRPLA) protein containing a pathological-length polyglutamine domain. ${ }^{137}$ In other studies, cystamine administration to

Table 2. List of polyglutamine (CAG-expansion) diseases.

\begin{tabular}{|c|c|c|c|c|c|}
\hline Disease & Sites of neuropathology & $\begin{array}{r}\text { CAG } \\
\text { nuI } \\
\text { Normal }\end{array}$ & $\begin{array}{l}\text { triplet } \\
\text { aber }\end{array}$ & $\begin{array}{c}\text { Gene product } \\
\text { (Intracellular } \\
\text { localization of protein } \\
\text { deposits) }\end{array}$ & Reference \\
\hline Corea Major or HD & Striatum (medium spiny neurons) and cortex in late stage & $6-35$ & $36-121$ & Huntingtin(n,c) & 108 \\
\hline SCAl & Cerebellar cortex (Purkinje cells), dentate nucleus and brain stem & $6-39$ & $40-81$ & Ataxin-1(n,c) & 109 \\
\hline SCA2 & Cerebellum, pontine nuclei, substantia nigra & $15-29$ & $35-64$ & Ataxin-2 (c) & 110 \\
\hline SCA3 or MJD & Substantia nigra, globus pallidus, pontine nucleus, cerebellar cortex & $13-42$ & 61-84 & Ataxin -3 (c) & 111 \\
\hline SCA6 & Cerebellar and mild brainstem atrophy & $4-18$ & $21-30$ & $\begin{array}{c}\text { Calcium channel } \\
\text { Subunit }(\alpha 1 \mathrm{~A})(\mathrm{m})\end{array}$ & 112 \\
\hline SCA7 & Photoreceptor and bipolar cells, cerebellar cortex, brainstem & $7-17$ & $37-130$ & Ataxin-7 (n) & 113 \\
\hline SCA12 & Cortical, cerebellar atrophy & $7-32$ & $41-78$ & $\begin{array}{c}\text { Brain specific regulatory } \\
\text { subunit of protein } \\
\text { phosphatase PP2A (?) }\end{array}$ & 114 \\
\hline SCA17 & Gliosis and neuronal loss in the Purkinje cell layer & $29-42$ & $46-637$ & TATA-binding protein (TBP) (n) & 115 \\
\hline SBMA or Kennedy & Motor neurons (anterior horn cells, bulbar neurons) & $11-34$ & $40-62$ & Androgen receptor (n, c) & 116 \\
\hline Disease & and dorsal root ganglia & & & & \\
\hline DRPLA & Globus pallidus, dentato-rubral and subthalamic nucleus & $7-35$ & $49-88$ & Atrophin (n, c) & 117 \\
\hline
\end{tabular}

HD, Huntington's Disease; SCA1, Spinocerebellar Ataxia Type 1; SCA2, Spinocerebellar Ataxia Type 2; SCA3, Spinocerebellar Ataxia Type 3; MJD, Machado-Joseph disease; SCA6, Spinocerebellar Ataxia Type 6; SCA7, Spinocerebellar Ataxia Type 7; SCA12, Spinocerebellar Ataxia Type 12; SCA17, Spinocerebellar Ataxia Type 17; SBMA, Spinobulbar Muscular Atrophy; DRPLA, Dentatorubral-pallidoluysian Atrophy. Cellular localization: c, cytosol; m, membrane; n, nucleus. 
HD-transgenic mice resulted in an increase in life expectancy and amelioration of neurological symptoms. ${ }^{138,139}$ Neuronal inclusions were decreased in one of these studies. ${ }^{139}$ Although all these scientific reports seem to support the hypothesis of a direct role of transglutaminase activity in the pathogenesis of the polyglutamine diseases, cystamine is also found to act in the HDtransgenic mice by mechanisms other than the inhibition of TGs, such as the inhibition of caspases, ${ }^{140}$ suggesting that this compound can have an additive effect in the therapy of HD. Currently, cysteamine is already in phase I studies in humans with HD, ${ }^{141}$ but several side effects, such as nausea, motor impairment and dosing schedule have been reported as reasons for nonadherence during phase II studies in human patients affected by cystinosis. ${ }^{142,143}$ Another critical problem in the use of TG inhibitors in treating neurological diseases relates to the fact that, as previously reported, the human brain contains at least four TGs, including TG1, 2, $3^{22}$ and TG6, ${ }^{144}$ and a strong non-selective inhibitor of TGs might also inhibit plasma Factor XIIIa, causing a bleeding disorder. Therefore, from a number of standpoints it would seem that a selective inhibitor, which discriminates between TGs, would be preferable to an indiscriminate $\mathrm{TG}$ inhibitor. In fact, although most of the TG activity in mouse brain, at least as assessed by an assay that measures the incorporation of radioactive putrescine (amine donor) into N,Ndimethyl casein (amine acceptor), seems to be due to TG2, ${ }^{145}$ no conclusive data have been obtained by TG2 gene knock-out experiments about the involvement of this TG in the development of the symptoms in HD-transgenic mice. ${ }^{26,146,147}$ Moreover, a recent scientific report showed that cystamine reduces aggregate formation in a mouse model of oculopharyngeal muscular dystrophy (OMPD), in which also the TG2 knockdown is capable of suppressing the aggregation and the toxicity of the mutant protein PABPN1, ${ }^{148}$ suggesting this compound as a possible therapeutic for OMPD.

\section{Conclusions}

In conclusion, numerous scientific reports have investigated aberrant TG activity both in cancer and in neurodegenerative diseases, but still today we are looking for experimental findings which could definitely confirm the direct involvement of TGs in the pathogenetic mechanisms responsible for these diseases. However, as result of the putative role of specific TG isoforms, such as TG2, in some human diseases, there is a considerable interest in developing inhibitors of these enzymes. Among those currently available, cystamine is the most commonly experimentally used to inhibit TG2 activity. In addition to cystamine, several types of TG2 inhibitors have been developed up to now. ${ }^{149}$ Interestingly, some of these inhibitors have shown promising results in experimental diabetic models. ${ }^{150}$ Therefore, the use of these inhibitors of TGs could be then useful also for other clinical approaches. To minimize the possible side effects, however, more selective inhibitors of the TGs should be required in the future. Progress in this area of research could be achieved, if possible, also through pharmaco-genetic approaches.

\section{References}

1. Folk JE. Mechanism and basis for specificity of transglutaminase-catalyzed $\varepsilon$-( $\gamma$-glutamyl) lysine bond formation. Adv Enzymol Relat Areas Mol Biol 1983;54:1-56.

2. Lorand L, Conrad S M. Transglutaminases. Mol Cell Biochem 1984;58:9-35.

3. Piacentini M, Martinet N, Beninati S, et al. Free and protein conjugatedpolyamines in mouse epidermal cells. Effect of high calcium and retinoic acid. J Biol Chem 1988;263:3790-4 .

4. Song Y, Kirkpatrick LL, Schilling AB, et al. Transglutaminase and polyamination of tubulin: posttranslational modification for stabilizing axonal microtubules. Neuron 2013; 78:109-23.

5. Achyuthan KE, Greenberg CS. Identification of a guanosine triphosphate-binding site on guinea pig liver transglutaminase. Role of GTP and calcium ions in modulating activity. J Biol Chem 1987;262:1901-6.

6. Hasegawa G, Suwa M, Ichikawa Y, et al. A novel function of tissue-type transglutaminase: protein disulfide isomerase. Biochem J 2003;373:793-803.

7. Lahav J, Karniel E, Bagoly Z, et al. Coagulation factor XIII serves as protein disulfide isomerase. Thromb Haemost 2009;101:840-4.

8. Iismaa SE, Mearns BM, Lorand L, et al. Transglutaminases and disease: lessons from genetically engineered mouse models and inherited disorders. Physiol Rev 2009;89:991-1023.

9. Smethurst PA, Griffin M. Measurement of tissue transglutaminase activity in a permeabilized cell system: its regulation by calcium and nucleotides. Biochem 1996;313:803-8.
10. Nakaoka H, Perez DM, Baek KJ, et al. Gh: a GTP-binding protein with transglutaminase activity and receptor signalling function. Science 1994;264: 1593-6.

11. Gentile V, Porta R, Chiosi E, et al. Tissue transglutaminase and adenylate cyclase interactions in Balb-C 3T3 fibroblast membranes. Biochim Biophys Acta 1997;1357:115-22.

12. Nanda N, Iismaa SE, Owens WA, et al. Targeted inactivation of $\mathrm{Gh} / \mathrm{tissue}$ transglutaminase II. J Biol Chem 2001;276:20673-8.

13. Mian S, El Alaoui S, Lawry J, et al. The importance of the GTP binding protein tissue transglutaminase in the regulation of cell cycle progression. FEBS Letters 1995;370:27-31.

14. Olaisen B, Gedde-Dahl TJR, Teisberg $\mathrm{P}$, et al. A structural locus for coagulation factor XIIIA (F13A) is located distal to the HLA region on chromosome $6 \mathrm{p}$ in man. Am J Hum Genet 1985;37:215-20.

15. Yamanishi K, Inazawa J, Liew F-M, et al. Structure of the gene for human transglutaminase 1. J Biol Chem 1992;267:17858-63.

16. Gentile V, Davies PJA, Baldini A. The human tissue transglutaminase gene maps on chromosome $20 \mathrm{q} 12$ by in situ fluorescence hybridization. Genomics 1994;20:295-7.

17. Wang M, Kim IG, Steinert PM, et al. Assignment of the human transglutaminase 2 (TGM2) and transglutaminase 3 (TGM3) genes to chromosome 20q11.2. Genomics 1994;23:721-2.

18. Gentile V, Grant F, Porta R, et al. Human prostate transglutaminase is localized on chromosome 3p21.33-p22 by in situ fluorescence hybridization. Genomics 1995;27:219-20.

19. Grenard P, Bates MK, Aeschlimann D. Evolution of transglutaminase genes: identification of a transglutaminases gene cluster on human chromosome 15q. Structure of the gene encoding transglutaminase $X$ and a novel gene family member, transglutaminase $Z$. J Biol Chem 2001;276:33066-78.

20. Thomas H, Beck K, Adamczyk M, et al. Transglutaminase 6: a protein associated with central nervous system development and motor function. Amino Acids 2013;44:161-77.

21. Bailey CDC, Johnson GVW. Developmental regulation of tissue transglutaminase in the mouse forebrain. J Neurochem 2004;91:1369-79.

22. Kim SY, Grant P, Lee JHC, et al. Differential expression of multiple transglutaminases in human brain. 
Increased expression and cross-linking by transglutaminase 1 and 2 in Alzheimer's disease. J Biol Chem 1999;274:30715-21.

23. lannaccone M, Giuberti G, De Vivo G, et al. Identification of a FXIIIA variant in human neuroblastoma cell lines. Int J Biochem Mol Biol 2013;4:102-7.

24. Citron BA, Santa Cruz KS, Davies PJ, et al. Intron-exon swapping of transglutaminase mRNA and neuronal tau aggregation in Alzheimer's disease. J Biol Chem 2001;276:3295-301.

25. De Laurenzi V, Melino G. Gene disruption of tissue transglutaminase. Mol Cell Biol 2001;21:148-55.

26. Mastroberardino PG, Iannicola C, Nardacci R, et al. 'Tissue' transglutaminase ablation reduces neuronal death and prolongs survival in a mouse model of Huntington's disease. Cell Death Differ 2002;9:873-80.

27. Lorand L, Graham RM. Transglutaminases: crosslinking enzymes with pleiotropic functions. Nature Mol Cell Biol 2003;4:140-56.

28. Wang Z, Griffin M. TG2, a novel extracellular protein with multiple functions. Amino Acids 2012;42:939-49.

29. Worthington JJ, Klementowicz JE, Travis MA. TGFbeta: a sleeping giant awoken by integrins. Trends Biochem Sci 2011;36:47-54.

30. Thiery JP, Acloque H, Huang RY, Nieto MA. Epithelial-mesenchymal transitions in development and disease. Cell 2009;139:871-90.

31. Telci D, Collighan RJ, Basaga H, Griffin M. Increased TG2 expression can result in induction of transforming growth factor beta1, causing increased synthesis and deposition of matrix proteins, which can be regulated by nitric oxide. J Biol Chem 2009;284:29547.

32. Lee J, Kim YS, Choi DH, et al. Transglutaminase 2 induces nuclear factor-kappaB activation via a novel pathway in BV-2 microglia. J Biol Chem 2004;279:53725-35.

33. Zemskov EA, Janiak A, Hang J, et al. The role of tissue transglutaminase in cell-matrix interactions. Front Biosci 2006;11:1057-76.

34. Zemskov EA, Loukinova E, Mikhailenko I, et al. Regulation of platelet-derived growth factor receptor function by integrin-associated cell surface transglutaminase. J Biol Chem 2009;284:16693-703.

35. Zemskov EA, Mikhailenko I, Smith EP, Belkin AM. Tissue transglutaminase promotes PDGF/PDGFR-mediated signaling and responses in vascular smooth muscle cells. J Cell Physiol
2012;227:2089-96.

36. Dardik R, Inbal A. Complex formation between tissue transglutaminase II (tTG) and vascular endothelial growth factor receptor 2 (VEGFR-2): proposed mechanism for modulation of endothelial cell response to VEGF. Exp Cell Res 2006;312:2973-82.

37. Antonyak MA, Li B, Regan AD, et al. Tissue transglutaminase is an essential participant in the epidermal growth factor-stimulated signaling pathway leading to cancer cell migration and invasion. $\mathrm{J}$ Biol Chem 2009;284:17914-25.

38. Walther DJ, Peter JU, Winter S, et al. Serotonylation of small GTPases is a signal transduction pathway that triggers platelet alpha-granule release. Cell 2003;115:851-62.

39. Guilluy C, Rolli-Derkinderen $M$, Tharaux PL, et al. Transglutaminasedependent RhoA activation and depletion by serotonin in vascular smooth muscle cells. J Biol Chem 2007;282:2918-28.

40. Beninati S, Nicolini L, Jakus J, et al. Identification of a substrate site for transglutaminases on the human protein synthesis initiation factor. Biochem J 1995;305:725-28.

41. Caraglia M, Passeggio A, Beninati S, et al. Interferon alpha 2 recombinant and epidermal growth factor modulate proliferation and hypusine synthesis in human epidermoid cancer KB cells. Biochem J 1997;324:737-41.

42. Caraglia M, Park MH, Wolff EC, et al. eIF5A isoforms and cancer: two brothers for two functions? Amino Acids 2013;44:103-9.

43. Giandomenico V, Lancillotti F, Fiorucci G, et al. Retinoic acid and IFN inhibition of cell proliferation is associated with apoptosis in squamous carcinoma cell lines: role of IRF-1 and TG II-dependent pathways. Cell Growth Differ 1997;8:91-100.

44. Lokshin A, Mayotte JA, Levitt ML. Mechanism of interferon-betainduced squamous differentiation and programmed cell death in human nonsmall-cell lung cancer cell lines. J Natl Cancer Inst 1995;87:206-12.

45. Nyman TA, Matikainen S, Sareneva T, et al. Proteome analysis reveals ubiquitin-conjugating enzymes to be a new family of interferon-alpha-regulated genes. Eur J Biochem 2000;267:401119.

46. Esposito C, Marra M, Giuberti G, et al. Ubiquitination of tissue transglutaminase is modulated by interferon alpha in human lung cancer cells. Biochem J
2003;370:205-12.

47. Begg GE, Holman SR, Stokes PH, et al. Mutation of a critical arginine in the GTP-binding site of transglutaminase 2 disinhibits intracellular cross-linking activity. J Biol Chem 2006;281:126039.

48. Park D, Choi SS, Ha KS Transglutaminase 2: a multi-functional protein in multiple subcellular compartments. Amino Acids 2010;39:61931.

49. McConoughey SJ, Basso M, Niatsetskaya ZV, et al. Inhibition of transglutaminase 2 mitigates transcriptional dysregulation in models of Huntington disease. EMBO Mol Med 2010:2:349-70.

50. Rodolfo C, Mormone E, Matarrese P, et al. Tissue transglutaminase is a multifunctional BH3-only protein. J Biol Chem 2004;279:54783-92.

51. Chowdhury ZA, Barsigian C, Chalupowicz GD, et al. Colocalization of tissue transglutaminase and stress fibers in human vascular smooth muscle cells and human umbilical vein endothelial cells. Exp Cell Res 1997;231:38-49.

52. Malorni W, Farrace MG, Matarrese P, et al. The adenine nucleotide translocator 1 acts as a type 2 transglutaminase substrate: implications for mitochondrial--dependent apoptosis. Cell Death Differ 2009;16:1480-92.

53. Birckbichler PJ, Patterson MK. Cellular transglutaminase, growth, and transformation. Ann NY Acad Sci 1978;312:354-65.

54. Beninati S, Abbruzzese A, Cardinali M. Differences in the post-translational modification of proteins by polyamines between weakly and highly metastatic B16 melanoma cells. Int J Cancer 1993;53:792-7.

55. Lentini A, Kleinman HK, Mattioli P, et al. Inhibition of melanoma pulmonary metastasis by methylxanthines due to decreased invasion and proliferation. Melanoma Res 1998;8:131-7.

56. Verderio EA, Johnson TS, Griffin M. Transglutaminases in wound healing and inflammation. Prog Exp Tumor Res 2005;38:89-114.

57. Luciani A, Villella VR, Esposito S, et al. Defective CFTR induces aggresome forformation and lung inflammation in cystic fibrosis through ROS-mediated autophagy inhibition. Nat Cell Biol 2010;12:863-75.

58. Park KS, Kim HK, Lee JH, et al. Transglutaminase 2 as a cisplatin resistance marker in non-small cell lung cancer. J Cancer Res Clin Oncol 
2010;136:493-502.

59. Verma A, Wang H, Manavathi B, et al. Increased expression of tissue transglutaminase in pancreatic ductal adenocarcinoma and its implications in drug resistance and metastasis. Cancer Res 2006;66:10525-33.

60. Quan G, Choi JY, Lee DS, Lee SC. TGF-beta1 up-regulates transglutaminase two and fibronectin in dermal fibroblasts: a possible mechanism for the stabilization of tissue inflammation. Arch Dermatol Res 2005;297:8490.

61. Suto N, Ikura K, Sasaki R. Expression induced by interleukin- 6 of tissue-type transglutaminase in human hepatoblastoma HepG2 cells. J Biol Chem 1993;268:7469-73.

62. Apte MV, Wilson JS. Dangerous liaisons: pancreatic stellate cells and pancreatic cancer cells. J Gastroenterol Hepatol 2012;27Suppl 2 69-74.

63. Shao M, Cao L, Shen C, et al. Epithelial-to-mesenchymal transition and ovarian tumor progression induced by tissue transglutaminase. Cancer Res 2009;69:9192-201.

64. Verma A, Guha S, Wang $H$, et al. Tissue transglutaminase regulates focal adhesion kinase/AKT activation by modulating PTEN expression in pancreatic cancer cells. Clin Cancer Res 2008;14:1997-2005.

65. Kumar A, Gao H, Xu J, et al. Evidence that aberrant expression of tissue transglutaminase promotes stem cell characteristics in mammary epithelial cells. PLoS One 2011;6:e20701.

66. Kumar A, Xu J, Brady S, et al. Tissue transglutaminase promotes drug resistance and invasion by inducing mesenchymal transition in mammary epithelial cells. PLoS One 2010 5:e13390.

67. Julien S, Puig I, Caretti E, et al. Activation of NF-kappaB by Akt upregulates Snail expression and induces epithelium mesenchyme transition. Oncogene 2007;26:7445-56.

68. Haase VH. Oxygen regulates epithelial-to-mesenchymal transition: insights into molecular mechanisms and relevance to disease. Kidney Int 2009;76:492-99.

69. Kaartineen MT, Pirhonen A, LinnalaKankkunen A, Maenpaa PH. Crosslinking of osteopontin by tissue transglutaminase increases its collagen binding properties. J Biol Chem 1999;274:1729-35.

70. Jones RA, Kotsakis P, Johnson TS, et al. Matrix changes induced by transglutaminase 2 lead to inhibition of angiogenesis and tumor growth. Cell Death Differ 2006; 13:1442-53.

71. Lentini A, Mattioli P, Provenzano B, et al. Role of the FAD-dependent polyamine oxidase in the selective formation of $\mathrm{N}(1), \mathrm{N}(8)$-bis(gamma-glutamyl)spermidine protein crosslinks(1). Biochem Soc Trans 2007;35:396-400.

72. Lentini A, Provenzano B, Caraglia M, et al. Impairment of the metastatic activity of melanoma cells by transglutaminase-catalyzed incorporation of polyamines into laminin and Matrigel. Amino Acids 2008;34:251-6.

73. Lentini A, Abbruzzese A, Caraglia M, et al. Protein-polyamine conjugation by transglutaminase in cancer cell differentiation: review article. Amino Acids 2004;26:331-7.

74. Carbone C, Di Gennaro E, Piro G, et al. Tissue transglutaminase (TG2) is involved in the resistance of cancer cells to the histone deacetylase (HDAC) inhibitor vorinostat. Amino Acids 2016;49:517-28.

75. Calcagno AM, Salcido CD, Gillet JP, et al. Prolonged drug selection of breast cancer cells and enrichment of cancer stem cell characteristics. J Natl Cancer Inst 2010;102:1637-52.

76. Erdem S, Yegen G, Telci D, et al. The increased transglutaminase 2 expression levels during initial tumorigenesis predict increased risk of metastasis and decreased disease-free and cancer-specific survivals in renal cell carcinoma. World J Urol 2015;33:1553-60.

77. Oh K, Ko E, Kim HS, et al. Transglutaminase 2 facilitates the distant hematogenous metastasis of breast cancer by modulating interleukin- 6 in cancer cells. Breast Cancer Res 2011;13:R96.

78. Jeong JH, Cho BC, Shim HS, et al. Transglutaminase 2 expression predicts progression free survival in nonsmall cell lung cancer patients treated with epidermal growth factor receptor tyrosine kinase inhibitor. J Korean Med Sci 2013;28:1005-14.

79. Block KI, Gyllenhaal C, Lowe L, et al. Designing a broad-spectrum integrative approach for cancer prevention and treatment. Semin Cancer Biol 2015;35:S276-304.

80. Samadi AK, Bilsland A, Georgakilas AG, et al. A multi-targeted approach to suppress tumor-promoting inflammation. Semin Cancer Biol 2015;35:S151-84.

81. Keillor JW, Apperley KY, Akbar A. Inhibitors of tissue transglutaminase. Trends Pharmacol Sci 2015;36:32-40.
82. Pietsch M, Wodtke R, Pietzsch J, Löser R. Tissue transglutaminase: an emerging target for therapy and imaging. Bioorg Med 2013;23:6528-43.

83. Huang S, Ingber DE. A non-genetic basis for cancer progression and metastasis: self-organizing attractions cell regulatory networks. Breast Disease 2007;26:27-54.

84. Hwang JY, Mangala LS, Fok JY, et al. Clinical and biological significance of tissue transglutaminase in ovarian carcinoma. Cancer Res 2008;68:5849-58.

85. Verma A, Guha S, Diagaradjane P, et al. Therapeutic significance of elevated tissue transglutaminase expression in pancreatic cancer. Clin Cancer Res 2008; 14:247.

86. Wolf J, Jäger C, Lachmann I, et al. Tissue transglutaminase is not a biochemical marker for Alzheimer's disease. Neurobiol Aging 2013;34:24958.

87. Wilhelmus MMM, Drukarch B. Tissue transglutaminase is a biochemical marker for Alzheimer's disease. Neurobiol Aging 2014;35:3-4.

88. Wolf J, Jäger C, Morawski M, et al. Tissue transglutaminase in Alzheimer's disease-facts and fiction: a reply to "Tissue transglutaminase is a biochemical marker for Alzheimer's disease". Neurobiol Aging 2014;35:5-9.

89. Adams RD, Victor M. Principles of neurology. New York: McGraw-Hill; 1993.

90. Selkoe DJ, Abraham C, Ihara Y. Alzheimer's disease: insolubility of partially purified paired helical filaments in sodium dodecyl sulfate and urea. Proc Natl Acad Sci USA 1982;79:6070-4.

91. Grierson AJ, Johnson GV, Miller CC. Three different human isoforms and rat neurofilament light, middle and heavy chain proteins are cellular substrates for transglutaminase. Neurosci Lett 2001;298:9-12.

92. Singer SM, Zainelli GM, Norlund MA. Transglutaminase bonds in neurofibrillary tangles and paired helical filament $\tau$ early in Alzheimer's disease. Neurochem Int 2002;40:17-30.

93. Halverson RA, Lewis J, Frausto S, et al. Tau protein is cross-linked by transglutaminase in $\mathrm{P} 301 \mathrm{~L}$ tau transgenic mice. J Neurosci 2005;25:1226-33.

94. Jeitner TM, Matson WR, Folk JE, et al. Increased levels of $\gamma$-glutamylamines in Huntington disease CSF. J Neurochem 2008;106:37-44.

95. Dudek SM, Johnson GV. Transglutaminase facilitates the formation of polymers of the beta-amyloid 
peptide. Brain Res 1994;651:129-33.

96. Hartley DM, Zhao C, Speier AC, et al. Transglutaminase induces protofibrillike amyloid $\beta$ protein assemblies that are protease-resistant and inhibit long-term potentiation. J Biol Chem 2008;283:16790-800.

97. Citron BA, Suo Z, SantaCruz K, et al. Protein crosslinking, tissue transglutaminase, alternative splicing and neurodegeneration. Neurochem Int 2002;40:69-78.

98. Junn E, Ronchetti RD, Quezado MM, et al. Tissue transglutaminase-induced aggregation of $\alpha$-synuclein: Implications for Lewy body formation in Parkinson's disease and dementia with Lewy bodies. Proc Natl Acad Sci USA 2003;100:2047-52.

99. Zemaitaitis MO, Lee JM, Troncoso JC, et al. Transglutaminase-induced crosslinking of $\mathrm{t}$ proteins in progressive supranuclear palsy. J Neuropathol Exp Neurol 2000;59:983-9.

100.Zemaitaitis MO, Kim SY, Halverson RA, et al. Transglutaminase activity, protein, and mRNA expression are increased in progressive supranuclear palsy. J Neuropathol Exp Neurol 2003;62:173-84.

101. Iuchi S, Hoffner G, Verbeke P, et al. Oligomeric and polymeric aggregates formed by proteins containing expanded polyglutamine. Proc Natl Acad Sci USA 2003;100:2409-14.

102. Gentile V, Sepe C, Calvani M, et al. Tissue transglutaminase-catalyzed formation of high-molecular-weight aggregates in vitro is favored with long polyglutamine domains: a possible mechanism contributing to CAGtriplet diseases. Arch Biochem Biophys 1998;352:314-21.

103. Kahlem P, Green H, Djian P. Transglutaminase action imitates Huntington's disease: selective polymerization of huntingtin containing expanded polyglutamine. Mol Cell 1998;1:595-601.

104. Karpuj MV, Garren H, Slunt H, et al. Transglutaminase aggregates huntingtin into nonamyloidogenic polymers, and its enzymatic activity increases in Huntington's disease brain nuclei. Proc Natl Acad Sci USA 1999;96:7388-93.

105. Segers-Nolten IM, Wilhelmus MM, Veldhuis $\mathrm{G}$, et al. Tissue transglutaminase modulates $\alpha$-synuclein oligomerization. Protein Sci 2008;17:1395-402.

106. Lai TS, Tucker T, Burke JR, et al. Effect of tissue transglutaminase on the solubility of proteins containing expanded polyglutamine repeats. J
Neurochem 2004;88:1253-60.

107. Konno T, Mori T, Shimizu H, et al. Paradoxical inhibition of protein aggregation and precipitation by transglutaminase-catalyzed intermolecular cross-linking. J Biol Chem 2005;280: 17520-5.

108. The Huntington's Disease Collaborative Research Group. A novel gene containing a trinucleotide repeat that is expanded and unstable on Huntington's disease chromosome. Cell 1993;72:971-83.

109. Banfi S, Chung MY, Jr KT, et al. Mapping and cloning of the critical region for the spinocerebellar ataxia type 1 gene (SCA1) in a yeast artificial chromosome contig spanning $1.2 \mathrm{Mb}$. Genomics 1993;18:627-35.

110. Sanpei K, Takano H, Igarashi S, et al. Identification of the spinocerebellar ataxia type 2 gene using a direct identification of repeat expansion and cloning technique, DIRECT. Nat Genet 1996;14:277-84.

111. Pujana MA, Volpini V, Estivill X. Large CAG/CTG repeat templates produced by PCR, usefulness for the DIRECT method of cloning genes with CAG/CTG repeat expansions. Nucleic Acids Res 1998;1:1352-3.

112. Fletcher CF, Lutz CM, O'Sullivan TN, et al. Absence epilepsy in tottering mutant mice is associated with calcium channel defects. Cell 1996;87:607-17.

113. Vincent JB, Neves-Pereira ML, Paterson AD, et al. An unstable trinucleotide-repeat region on chromosome 13 implicated in spinocerebellar ataxia: a common expansion locus. Am J Hum Genet 2000;66:819-29.

114. Holmes SE, O'Hearn E, Margolis RL. Why is SCA12 different from other SCAs? Cytogenet Genome Res 2003;100:189-97.

115. Imbert G, Trottier Y, Beckmann J, et al. The gene for the TATA binding protein (TBP) that contains a highly polymorphic protein coding CAG repeat maps to 6q27. Genomics 1994;21:667-8.

116. La Spada AR, Wilson EM, Lubahn DB, et al. Androgen receptor gene mutations in X-linked spinal and bulbar muscular atrophy. Nature 1991;352:77-9.

117. Onodera O, Oyake M, Takano H, et al. Molecular cloning of a full-length cDNA for dentatorubral-pallidoluysian atrophy and regional expressions of the expanded alleles in the CNS. Am J Hum Genet 1995;57:1050-60.

118. Cooper AJL, Sheu K-FR, Burke JR, et al. Pathogenesis of inclusion bodies in $(\mathrm{CAG}) \mathrm{n} / \mathrm{Qn}$-expansion diseases with special reference to the role of tissue transglutaminase and to selective vulnerability. J Neurochem 1999;72:88999.

119. Hadjivassiliou M, Maki M, Sanders DS, et al. Autoantibody targeting of brain and intestinal transglutaminase in gluten ataxia. Neurology 2006;66:3737.

120. Boscolo S, Lorenzon A, Sblattero D, et al. Anti transglutaminase antibodies cause ataxia in mice. Plos One 2010;5:e9698.

121. Stamnaes J, Dorum S, Fleckenstein B, et al. Gluten $\mathrm{T}$ cell epitope targeting by TG3 and TG6; implications for dermatitis herpetiformis and gluten ataxia. Amino Acids 2010;39:1183-91.

122. Lerner A, Matthias T. GUT-the Trojan horse in remote organs' autoimmunity. J Clin Cell Immunol 2016; 7: 401.

123. Matthias T, Jeremias P, Neidhofer S, et al. The industrial food additive microbial transglutaminase, mimics the tissue transglutaminase and is immunogenic in celiac disease patients. Autoimmun Rev 2016;15:1111-9.

124. Lerner A, Neidhofer S, Matthias T. Transglutaminase 2 and anti transglutaminase 2 autoantibodies in celiac disease and beyond: Part A: TG2 doubleedged sword: gut and extraintestinal involvement. Immunome Res 2015; 11 : 101-5.

125. Wakshlag JJ, Antonyak MA, Boehm JE, et al. Effects of tissue transglutaminase on beta-amyloid 1-42-induced apoptosis. Protein J 2006;25:83-94.

126. Lee JH, Jeong J, Jeong EM, et al. Endoplasmic reticulum stress activates transglutaminase 2 leading to protein aggregation. Int J Mol Med 2014;33: 849-55.

127. Grosso H, Woo JM, Lee KW, et al. Transglutaminase 2 exacerbates $\alpha$ synuclein toxicity in mice and yeast. FASEB J 2014;28:4280-91.

128. Zhang J, Wang S, Huang W, et al. Tissue transglutaminase and its product isopeptide are increased in Alzheimer's disease and APPswe/PS1dE9 double transgenic mice brains. Mol Neurobiol 2016;53:5066-78.

129. Wilhelmus MM, De JM, Smit AB, et al. Catalytically active tissue transglutaminase colocalises with $\mathrm{A} \beta$ pathology in Alzheimer's disease mouse models. Sci Rep 2016;6:20569.

130. Wilhelmus MMM, De JM, Rozemuller AJM, et al. Transglutaminase 1 and its regulator Tazarotene-induced gene 3 localize to neuronal tau inclusions in tauopathies. J Pathol 2012;226:132-42. 
131. Basso M, Berlin J, Xia L, et al. Transglutaminase inhibition protects against oxidative stress-induced neuronal death downstream of pathological ERK activation. J Neurosci 2012;39:6561-9.

132. Lee J, Kim YS, Choi DH, et al. Transglutaminase 2 induces nuclear factor-kB activation via a novel pathway in BV-2 microglia. J Biol Chem 2004;279:53725-35.

133. Kumar S, Mehta K. Tissue transglutaminase constitutively activates HIF-1a promoter and nuclear factor-kB via a non-canonical pathway. Plos One 2012; 7:e49321.

134. Lu S, Saydak M, Gentile V, et al. Isolation and characterization of the human tissue transglutaminase promoter. J Biol Chem 1995;270:9748-55.

135. Ientile R, Currò $M$, Caccamo D Transglutaminase 2 and neuroinflammation. Amino Acids 2015;47:19-26.

136. Griffith OW, Larsson A, Meister A. Inhibition of $\gamma$-glutamylcysteine synthetase by cystamine: an approach to a therapy of 5-oxoprolinuria (pyroglutamic aciduria). Biochem Biophys Res Commun 1977;79:919-25.

137. Igarashi S, Koide R, Shimohata T, et al. Suppression of aggregate formation and apoptosis by transglutaminase inhibitors in cells expressing truncated DRPLA protein with an expanded polyglutamine stretch. Nat Genet 1998;18:111-7.
138. Karpuj MV, Becher MW, Springer JE, et al. Prolonged survival and decreased abnormal movements in transgenic model of Huntington disease, with administration of the transglutaminase inhibitor cystamine. Nat Med 2002;8:143-9.

139. Dedeoglu A, Kubilus JK, Jeitner TM, et al. Therapeutic effects of cystamine in a murine model of Huntington's disease. J Neurosci 2002;22:8942-50.

140. Lesort M, Lee M, Tucholski J, et al. Cystamine inhibits caspase activity. Implications for the treatment of polyglutamine disorders. J Biol Chem 2003;278:3825-30.

141. Dubinsky R, Gray C. CYTE-I-HD: Phase I Dose Finding and Tolerability Study of Cysteamine (Cystagon) in Huntington's Disease. Movement Disord 2006;21:530-3.

142. Langman CB, Greenbaum LA, Sarwal $\mathrm{M}$, et al. A randomized controlled crossover trial with delayed-release cysteamine bitartrate in nephropathic cystinosis: effectiveness on white blood cell cystine levels and comparison of safety. Clin J Am Soc Nephrol 2012;7:1112-20.

143. Besouw M, Masereeuw R, Van DHL, et al. Cysteamine: an old drug with new potential. Drug Discov Today 2013;18:785-92.

144. Hadjivassiliou M, Aeschlimann P, Strigun A, et al. Autoantibodies in gluten ataxia recognize a novel neu- ronal transglutaminase. Ann Neurol 2008;64:332-43.

145. Krasnikov BF, Kim SY, McConoughey $\mathrm{SJ}$, et al. Transglutaminase activity is present in highly purified nonsynaptosomal mouse brain and liver mitochondria. Biochemistry 2005;44:7830-43.

146. Menalled LB, Kudwa AE, Oakeshott S, et al. (2014) Genetic deletion of transglutaminase 2 does not rescue the phenotypic deficits observed in R6/2 and zQ175 mouse models of Huntington's disease. Plos One 2014;9:e99520.

147. Bailey CD, Johnson GV. Tissue transglutaminase contributes to disease progression in the R6/2 Huntington's disease mouse model via aggregate-independent mechanisms. J Neurochem 2005;92:83-92.

148. Davies JE, Rose C, Sarkar S, et al. Cystamine suppresses polyalanine toxicity in a mouse model of oculopharyngeal muscular dystrophy. Sci Transl Med 2010;2:34-40.

149. Pietsch M, Wodtke R, Pietzsch J, et al. Tissue transglutaminase: An emerging target for therapy and imaging. Bioorg Med Chem Lett 2013;23:6528-3.

150. Bhatt MP, Lim YC, Hwang J, et al. Cpeptide prevents hyperglycemiainduced endothelial apoptosis through inhibition of reactive oxygen speciesmediated transglutaminase 2 activation. Diabetes 2013;62:243-53. 